\title{
CDF Status and B Physics Prospects
}

\author{
J. Stephen Miller a for the CDF collaboration

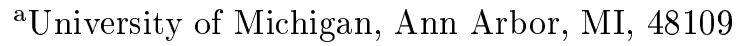

With extensive upgrades to the detector and electronics, CDF has started collecting data with Run II of the Tevatron. The enhanced silicon coverage and the use of silicon based tracks in the trigger, make CDF well suited for a broad program of B hadron measurements. We present the current status of the experiment and prospects for measurements in Run II.

\section{Introduction}

The Tevatron accelerator and the Collider Detector at Fermilab (CDF) have had extensive upgrades and are now collecting data as part of Run II of the Tevatron. The large cross section for $b$ quark production in $p \bar{p}$ collisions at the Tevatron gives a high production rate of $b$ hadrons. The upgrades to the detector and electronics allows CDF to collect a large sample of events and pursue a broad program of $b$ physics measurements. These include measurements of $\mathrm{CP}$ violation using modes such as $B \rightarrow J / \psi K_{s}, B \rightarrow \pi \pi$, $B_{s} \rightarrow K K$, and $B_{s} \rightarrow D_{s} K ; B_{s}$ mixing; searches for rare $B$ decays as well as measuring the lifetimes, masses and branching ratios for many $b$ hadrons. The measurements involving $B_{s}, B_{c}$, and $\Lambda_{b}$ hadrons are unique to the Tevatron and nicely complement measurements made at the $\mathrm{B}$ factories.

We describe the current status of the detector, the trigger strategy for collecting large samples of $B$ and charm mesons, and some of the physics measurements that will be made. A review of Run I measurements and an overview of the $B$ physics program at the Tevatron is described in a separate contribution to these proceedings [1].

\section{Detector Status}

For the upgrade [2] for Run II, much of the CDF detector has been replaced with only the solenoid, central calorimeter and central and extension muon detectors being retained. The new detector has more silicon layers and increased sil- icon coverage, enhancing the ability for reconstructing displaced vertices from $B$ decays. The addition of a time of flight system which is combined with $\mathrm{d} E / \mathrm{d} x$ measurements improves particle identification which is critical for flavor tagging. All new readout and trigger electronics was built. This change was required to accommodate the Tevatron upgrade from $6 \times 6$ bunches with a 3.5 $\mu s$ spacing to $36 \times 36$ bunches with $396 \mathrm{~ns}$ spacing and a possible further upgrade to $132 \mathrm{~ns}$ bunch spacing. The trigger and DAQ bandwidth was increased to handle higher luminosities and extend CDF's physics capabilities, especially in the area of $B$ physics.

\subsection{Detector Systems}

The silicon tracker has three parts: Layer00, SVXII, and the Intermediate Silicon Layer (ISL). Layer 00 is a single layer of r-phi measurements $1.4 \mathrm{~cm}$ from the beamline. This layer provides improved impact parameter resolution which is particularly useful for measuring $B_{s}$ mixing. SVXII has five double sided layers with r-phi measurements on one side. The other side measures $r-z$ hits on three layers and has small angle stereo on two of the layers. The 5 r-phi layers of SVXII are used for triggering on SVX tracks with the L2 trigger. With a length of $87 \mathrm{~cm}$, the silicon tracker covers the luminous region of the $p \bar{p}$ collisions. The ISL has both r-phi and small angle stereo strips. There is one layer in the central region of $|\eta|<1$ and 2 layers for $1<|\eta|<2$. The ISL provides better matching between drift chamber tracks and the SVX tracks. In addition, 
it will allow for standalone silicon tracking out to larger angles than is allowed by the drift chamber.

The central drift chamber (COT) has $8 \mathrm{su}-$ perlayers with each superlayer having 12 sense wires per phi slice. The superlayers alternate between axial and small angle stereo measurements. The cell size is significantly smaller than in the Run I drift chamber because of the need for the drift time to be less than the $132 \mathrm{~ns}$ beam crossing period for the upgraded Tevatron. The drift time is read out with multi-hit TDC's along with pulse height information for $\mathrm{d} E / \mathrm{d} x$ measurements. The hit resolution is $175 \mu \mathrm{m}$ and the tracking efficiency is $99.7 \%$ for high $p_{t}$ tracks. Reconstructed tracks combine both COT and SVX hits and give single track impact parameter resolutions of $23 \mu \mathrm{m}$. The decay distance resolution measured using muons from $J / \psi$ decays is about $60 \mu \mathrm{m}$ including $35 \mu \mathrm{m}$ for the beam spot resolution. Further improvements to the alignment and the addition of the innermost silicon layer hits (L00), will improve this performance.

Just outside the drift chamber is a time of flight detector using scintillator bars with a $4 \times 4 \mathrm{~cm}^{2}$ cross section. This detector is new for Run II and provides enhanced capability for particle identification for flavor tagging. Preliminary calibrations give a time resolution of $110 \mathrm{ps}$ which is close to the goal of $100 \mathrm{ps}$. At that resolution there is $2 \sigma K \pi$ separation for tracks with momentum less than $1.6 \mathrm{GeV} / \mathrm{c}$.

The central calorimeter is the same one used in Run I except for all new readout electronics to accommodate the much shorter bunch spacing in Run II. This detector has a tower geometry with each tower covering $15^{\circ}$ in phi and 0.11 in eta. Each tower has both an electromagnetic section of scintillator and lead and a hadronic section using scintillator and iron. In the electromagnetic section at the position of shower maximum is a 2-d strip and wire chamber for improved electron and photon identification. An all new plug calorimeter also using lead and iron plus scintillator replaced the Run I gas calorimeter. The central muon chambers have new readout electronics plus some additional chambers to cover some small gaps that existed in the muon coverage. The new forward muon detectors provide

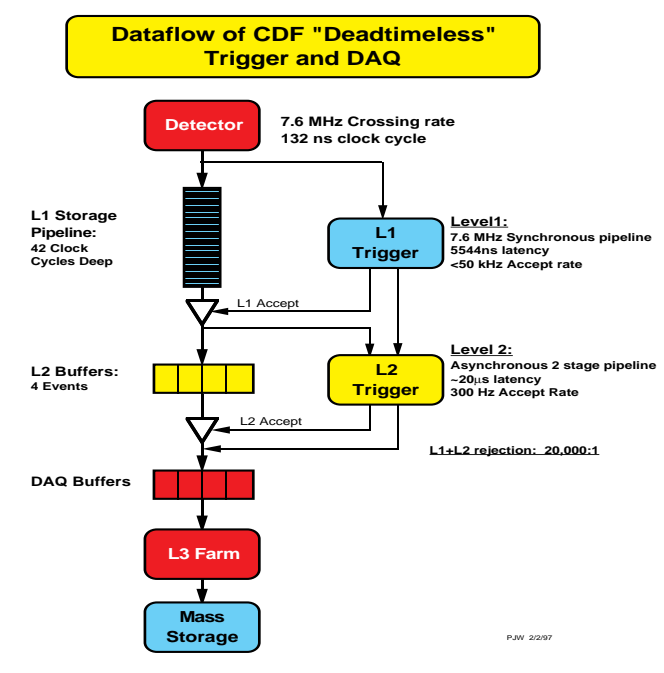

Figure 1. Diagram of the CDF trigger architecture.

coverage out to $\eta<2$ for identifying muons, and extends the trigger coverage to $\eta<1.5 \mathrm{~A}$ new luminosity monitor measures the accelerator luminosity with an uncertainty of less than $5 \%$.

\subsection{Trigger and DAQ}

The L1 trigger is a deadtimeless trigger with a 42 stage pipeline and can make a trigger decision every $132 \mathrm{~ns}$ with a total pipeline latency of 5544 ns. L1 uses information from the electromagnetic and hadronic parts of the calorimeter towers, the total transverse energy, missing transverse energy in the calorimeter, hits in the muon chambers, and tracks with $p_{t}>1.5 \mathrm{GeV} / \mathrm{c}$ using COT axial layer hits. The tracks can be matched to the calorimeter and muon chambers for electron and muon triggers. In addition, a trigger requiring only 2 tracks at L1 is used as the first stage of a hadronic $B$ trigger. This trigger dominates the rate of L1 accepts. The addition of tracking at L1 is a significant improvement over the Run I trigger.

The L2 trigger adds information to the objects found by the L1 trigger. Calorimeter towers are grouped into clusters for jet, electron and photon triggers and calorimeter energy isolation 
quantities are calculated. Hits from the shower maximum detectors inside the electromagnetic calorimeter are used for better track matching for electron triggers. The full list of tracks found at L1 are also available. The most innovative addition to the L2 trigger is the silicon vertex trigger (SVT) which provides $r-\phi$ silicon based tracks for the L2 trigger decision [3]. The ability to trigger on silicon based tracks at a hadron collider is a new capability which significantly enhances CDF's $B$ and charm physics programs. This is possible because the SVX readout chip stores the hit information every $132 \mathrm{~ns}$ and has fast digitization and readout after a L1A. The L1 COT based tracks define roads for finding silicon hits which are matched to patterns of hits in lookup tables to find the SVT tracks. The tracks are fit with resolutions of $\sigma d_{0} \approx 35 \mu \mathrm{m}, \sigma_{\text {curvature }} \approx 6 x 10^{-6} \mathrm{~cm}^{-1}$ and $\sigma \phi_{0} \approx 1 \mathrm{mrad}$. The L2 trigger decision is made inside custom built processor boards running $\mathrm{C}++$ algorithms which make simple cuts as well as calculate displaced vertices and transverse masses. The L2 trigger will need to handle a L1 accept rate of $30-40 \mathrm{kHz}$ at the highest luminosities and outputs a L2A rate of $300 \mathrm{~Hz}$.

After a L2 trigger accept, the DAQ reads out all the information from the detector and sends it to a farm of 240 dual processor PC's. These processors run the standard offline code and do full event reconstruction for making the final selection. This gives an output rate of $50 \mathrm{~Hz}$ which is stored on tape.

\subsection{B Physics Trigger Strategy}

The initial challenge of $B$ physics measurements at the Tevatron is triggering on $b$ quark events from a total cross section for light quark production that is three orders of magnitude larger. In addition, the large $\mathrm{b}$ production cross section (and total cross section) requires a large trigger bandwidth to handle the resulting high trigger rates.

$B$ hadron events are selected using three general kinds of triggers. These are the hadronic trigger, which uses tracks with large impact parameters; the lepton plus displaced track trigger; and the di-lepton trigger which predominantly selects events with $J / \psi$ decays.
The hadronic trigger exploits the long lifetime of $B$ hadrons to select fully hadronic $B$ decays. This trigger has a very loose requirement at L1 of 2 oppositely charged tracks with transverse momentum greater than $2 \mathrm{GeV} / \mathrm{c}$. The L2 trigger uses SVT based tracks and requires that the tracks have an impact parameter greater than 100 $\mu \mathrm{m}$ as well as a displaced vertex for the 2 tracks. This trigger selects rare two body hadronic $B$ decays such as $B \rightarrow \pi \pi(K K)$ used for $\mathrm{CP}$ violating measurements, as well as hadronic $B_{s}$ decays which have the best time resolution for measuring $B_{s}$ mixing. This triggering capability did not exist during Run I and has greatly extended the $B$ physics capabilities of CDF. Figure 2 illustrates a sample of $D \rightarrow K \pi \pi$ events selected with the hadronic trigger.

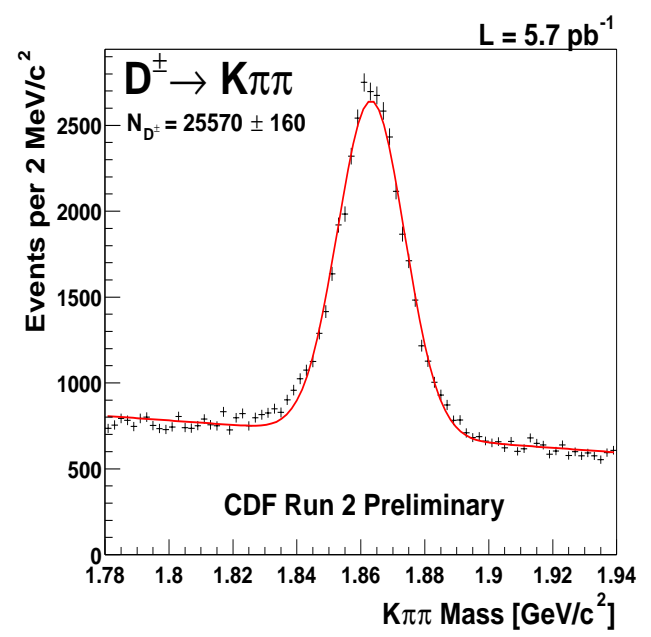

Figure 2. Reconstructed $D^{+} \rightarrow K \pi \pi$ mass from events selected using the hadronic track trigger.

The lepton plus displaced track trigger requires an electron or muon with a $p_{t}$ greater than 4 $\mathrm{GeV} / \mathrm{c}$ plus an additional track with a large impact parameter. Requiring the displaced track allows a low lepton momentum threshold and increases the yield of semileptonic decays by a fac- 
tor of 3 over what was obtained in Run I using a trigger which required a single lepton with $p_{t}$ greater than $8 \mathrm{GeV} / \mathrm{c}$. This trigger selects semileptonic $B$ decays which provide a sample useful for many $B$ measurements including fragmentation fractions and lifetimes of $B^{+}, B^{0}, B_{s}$, and $\Lambda_{b}$ hadrons, $b$ flavor tagging studies and $B$ mixing measurements. In addition, radiative $B$ decays such as $B \rightarrow K^{*} \gamma$ and $B_{s} \rightarrow \phi \gamma$ can be selected in cases where the photon converts to an electron-positron pair.

Dilepton events are selected either with two oppositely charged muons with $p_{t}$ greater than $1.5 \mathrm{GeV} / \mathrm{c}$ or two oppositely charged electrons with $p_{t}$ greater than $2 \mathrm{GeV} / \mathrm{c}$. These thresholds are lower than those used in Run I and the yield of $J / \psi$ events is 2 to 3 times higher. $B \rightarrow J / \psi X$ events are used for measuring $\sin 2 \beta$ in $B \rightarrow J / \psi K_{s}$ decays, and measuring exclusive $B$ meson lifetimes. Di-lepton events are also used for searches for electroweak penguin decays such as $B \rightarrow K^{(*)} \ell^{+} \ell^{-}$decays and rare $B_{(s)} \rightarrow \ell^{+} \ell^{-}$ decays.

\section{Charm Physics Prospects}

The hadronic $B$ trigger, using displaced tracks, selects a large sample of hadronic charm decays. The expected yields for $50 \mathrm{pb}^{-1}$ are given in Table 1 . With the $2 \mathrm{fb}^{-1}$ expected for the first part of Run II, the charm samples will be 10 to 100 times those of the charm fixed target experiments. This large data sample will allow a significant charm physics program.

Direct charm production can be separated from charm from $B$ meson decay by looking at the impact parameter of the charm meson. For $D$ 's from direct production the reconstructed $D$ meson flight direction points back to the primary vertex. In contrast, $D$ 's from $B$ decay will tend to have a large impact parameter with respect to the primary vertex. The resolution of the reconstructed $D$ impact parameter is about $40 \mu \mathrm{m}$.

CDF is measuring the differential production cross sections for $D^{0}, D^{+}, D^{*+}, D_{s}$, and $\Lambda_{c}$ hadrons. Both $D^{+}$and $D_{s}$ are reconstructed in the final state $\phi(K K) \pi$, as shown in Figure 3, giving a precise measurement of the $D_{s}-D^{+}$mass
Table 1

The expected yield of hadronic charm decays with $50 \mathrm{pb}^{-1}$

\begin{tabular}{ll}
\hline$D$ Decay Mode & Events \\
\hline$D^{0} \rightarrow K \pi$ & $300 \mathrm{~K}$ \\
$D^{0} \rightarrow K K$ & $20 \mathrm{~K}$ \\
$D^{0} \rightarrow \pi \pi$ & $10 \mathrm{~K}$ \\
$D^{+} \rightarrow K \pi \pi$ & $200 \mathrm{~K}$ \\
$D_{s} \rightarrow K K \pi$ & $10 \mathrm{~K}$ \\
$D^{*+} \rightarrow D^{0} \pi\left(D^{0} \rightarrow K \pi\right)$ & $80 \mathrm{~K}$ \\
$\Lambda_{c} \rightarrow p K \pi$ & 300 \\
\hline
\end{tabular}

difference.

Searches for direct CP violation can be done by comparing decay widths of $\mathrm{CP}$ conjugate decay modes. Charged modes such as $D^{+} \rightarrow K^{+} K^{-} \pi^{+}$ and neutral modes such as $D^{0} \rightarrow K^{+} K^{-}$and $D^{0} \rightarrow \pi^{+} \pi^{-}$can both be used. The large data sample will also give strong limits on rare charm decays such as $D^{+} \rightarrow \pi^{+} \mu^{+} \mu^{-}$. In addition, limits on $D^{0}-\bar{D}^{0}$ mixing can be placed by measuring the lifetime difference between different $\mathrm{CP}$ final states, and looking at wrong sign hadronic and semileptonic decays.

\subsection{B Physics Prospects}

With all the detector and trigger components needed for $B$ physics measurements working well, CDF will make a large number of interesting measurements. These include measurements of lifetimes, masses and branching ratios for many $b$ hadrons, $B$ mixing, $\mathrm{CP}$ violation and searches for rare and forbidden decays [4]. Below we discuss in more detail just a few of these measurements.

The hadronic trigger selects $B_{s}$ decay modes such as $B_{s} \rightarrow D_{s} \pi$ and $B_{s} \rightarrow D_{s} \pi \pi \pi$ with $D_{s} \rightarrow \phi(K K) \pi$. These can be used for measuring the $B_{s}$ mixing parameter $x_{s}=\Delta m_{s} / \Gamma_{s}$. Fully reconstructed $B_{s}$ decays provide good resolution measurement of the proper time for the decay since the $B_{s}$ momentum is directly measured. Using all silicon layers we expect a proper time resolution of about $45 \mathrm{fs}$. The flavor of the $B_{s}$ at decay is given by the reconstructed final state. In order to measure $B_{s}$ mixing, the fla- 


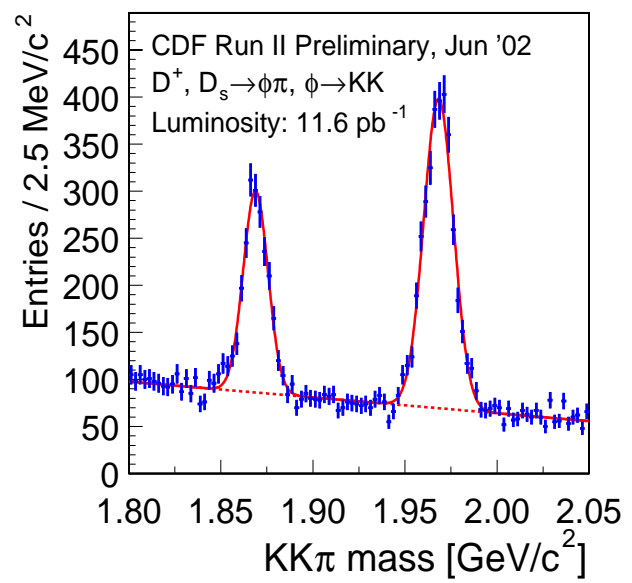

Figure 3. Reconstructed $D^{+}$and $D_{s}$ candidate mass for the decay mode $\phi(K K) \pi$.

vor of the $B_{s}$ at production must be measured. Since the $b$ quarks are produced in pairs, identifying the flavor of the $b$ quark opposite the $B_{s}$ gives the flavor of the $B_{s}$ at production. There are several opposite side flavor tagging techniques which include: using the charge of the lepton from semileptonic $B$ decay, measuring the total charge using charged tracks in the jet, and identifying the kaon which comes from the sequential $D$ decay. One can also look at the charge of fragmentation tracks which are associated with the $B_{s}$ production. This same side tagger uses time of flight and $\mathrm{d} E / \mathrm{d} x$ to identify the fragmentation $K$ associated with the $B_{s}$. Same side pion tags can be used for flavor tagging $B_{d}^{0}$ events. Two important numbers for flavor taggers are the efficiency $(\epsilon)$ for finding the tag and the mistag rate $(w)$, which is the fraction of events where the wrong flavor was determined. The mistag rate is often defined as a dilution factor $D=1-2 w$. The uncertainty on $x_{s}$ is proportional to $1 / \sqrt{\epsilon D^{2}}$. Using all of the tagging methods mentioned, we expect a value of $11.3 \%$ for $\epsilon D^{2}$. CDF should be able to measure $x_{s}$ for $x_{s}<60$ [4]

The $B_{s}$ lifetime difference $\Delta \Gamma_{s}$ can be mea- sured using the decay $B_{s} \rightarrow J / \psi \phi[5]$. That decay mode has both $\mathrm{CP}$ even and odd states which can be separated using the decay angular distributions of the decay. By doing a combined fit to the lifetime and decay angles, a more precise measurement can be made than simply fitting the lifetime to two exponentials. We expect an error on $\Delta \Gamma_{s} / \Gamma_{s}$ of $0.05-0.08$.

A search for $\mathrm{CP}$ violation in the decay $B_{s} \rightarrow$ $J / \psi \phi$ is interesting because the asymmetry in the Standard Model is extremely small. Any observation of an asymmetry above a few percent would be a clear signal for physics beyond the Standard Model. This analysis would also use the angular distributions to separate the $\mathrm{CP}$ even and odd states. We expect an error of 0.1 to 0.2 on the asymmetry, depending on the value of $x_{s}$.

\section{Conclusion}

The CDF detector is performing very well and has been collecting data as part of Run II of the Tevatron. With the new capability of using silicon based tracks in the trigger, large samples of $B$ and charm mesons are being collected. A broad physics program of charm and $B$ measurements will be made, furthering our understanding of heavy flavour properties and the CKM matrix.

\section{REFERENCES}

1. J. Cranshaw, these proceedings.

2. R. Blair et al, [the CDF collaboration], FERMILAB-Pub-96/390-E (1996).

3. W. Ashmanskas et al., FERMILAB-CONF02-035-E, (2002)

4. K. Anikeev et al., FERMILAB-Pub-01/197 (2001).

5. A. S. Dighe, I. Dunietz, H. J. Lipkin, and J. L. Rosner, Phys. Lett. B369, 144 (1996). 